Originalien

Nervenarzt $2021 \cdot 92: 27-35$

https://doi.org/10.1007/s00115-020-00957-2

Online publiziert: 17 . Juli 2020

(c) Der/die Autor(en) 2020

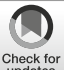

\author{
Johannes Fuß ${ }^{1} \cdot$ Inga Marquardt ${ }^{2} \cdot$ Peer Briken ${ }^{1}$. Norbert Konrad ${ }^{3}$ \\ 'Institut für Sexualforschung, Sexualmedizin und Forensische Psychiatrie, Zentrum für Psychosoziale \\ Medizin, Universitätsklinikum Hamburg-Eppendorf, Hamburg, Deutschland \\ ${ }^{2}$ Amtsgericht Hamburg, Hamburg, Deutschland \\ ${ }^{3}$ Institut für Forensische Psychiatrie, Charité - Universitätsmedizin Berlin, Berlin, Deutschland
}

\title{
Zwangsmedikation psychisch erkrankter Menschen im Justizvollzug
}

\section{Situation in Deutschland und Empfehlungen für die Praxis}

\section{Hintergrund}

In Westeuropa und den USA wird seit über 80 Jahren über einen Zusammenhang zwischen der Zahl stationärpsychiatrischer Betten und der Zahl an Gefängnisinsassen diskutiert. Wenn psychiatrische Betten abgebaut werden, komme - so die als Penrose-Hypothese bekannt gewordene Theorie [1] - ein Teil der zuvor stationär behandelten Menschen früher oder später in Gefängnissen oder forensisch-psychiatrischen Einrichtungen unter. Die Theorie wurde immer wieder kritisiert und relativiert, jedoch untermauern mehrere longitudinale Studien [2-4], dass der zunehmende Abbau allgemeinpsychiatrischer Betten und die Verkürzung der Liegezeiten im Zuge der Deinstitutionalisierung mit einer drastischen Zunahme der Betten in forensisch-psychiatrischen Krankenhäusern und Gefängnissen einherging. ${ }^{1}$ Eine Schlussfolgerung aus der PenroseHypothese könnte sein, dass ein Teil der psychisch erkrankten Menschen ein stark strukturiertes Setting benötigen, das ihre Medikamenteneinnahme sicherstellt und Kontrollfunktionen übernimmt. Für Deutschland ist diese Entwicklung erst seit Mitte der 1990er-Jahre festzustellen [5]. Die angenommene Ver-

1 Diese Entwicklung wurde auch als Reinstitutionalisierung oder Transinstitutionalisierung bezeichnet. lagerung schwer erkrankter Patienten von stationären Allgemeinpsychiatrien in Gefängnisse [6] führte zu kritischen Leitartikeln wie „Bring back the asylum“ [7], „Does deinstitutionalization cause criminalization“ [8]? oder „Prisons as new asylums“" [9]. Gegenpositionen (z. B. [10]) postulieren, dass auch voneinander unabhängige Entwicklungen die gegenläufigen Belegungstrends in der Allgemeinpsychiatrie und forensischen Psychiatrie bzw. im Justizvollzug erklären können. Einigkeit dürfte aber darüber bestehen, dass psychische Erkrankungen in Gefängnissen schlechter behandelt werden können als in psychiatrischen Einrichtungen. Trotzdem kommen psychische Erkrankungen bei Menschen im Justizvollzug häufig vor [11-13]. Möglicherweise hat die Zahl schwer erkrankter Patienten, die zusätzlich oft obdachlos, alleinstehend und arbeitslos sind [14], in deutschen Gefängnissen zugenommen. In der Berliner Gefängnispsychiatrie waren Ende Januar 2020 über $80 \%$ der Patienten obdachlos, gegenüber ca. $13 \%$ der Patienten in allgemeinpsychiatrischen Versorgungskliniken [15]. Obwohl Längsschnittstudien für Deutschland fehlen, kann bei aktuell circa 50.000 inhaftierten Strafgefangenen [16] und circa 15.000 Untersuchungshaftgefangenen [17] davon ausgegangen werden, dass mindestens 2500 Menschen in Haft unter Psychosen leiden - die Zahl an Menschen mit affektiven
Erkrankungen dürfte deutlich höher liegen. Diese „Forensifizierung“ schwer kranker Patienten dürfte auch begünstigt haben, dass die Rechtsprechung die $\mathrm{zu}$ begründenden Voraussetzungen für eine Zwangsmedikation außerhalb des Justizvollzuges in den letzten Jahren konkretisiert und dadurch erhöht hat. Dies dürfte eine Abnahme der Zwangsmedikationen zur Folge gehabt haben [18]. ${ }^{2} \quad$ Ein Zusammenhang zwischen unterlassener Zwangsmedikation und der Zunahme aggressiver Übergriffe ist wissenschaftlich gut belegt [19]. Dies lässt die Vermutung $\mathrm{zu}$, dass einzelne psychisch erkrankte Menschen aufgrund einer unterlassenen Zwangsmedikation straffällig geworden sind und so in den Justizvollzug gelangten. Auch in $\mathrm{Zu}$ kunft ist daher zu befürchten, dass bei gleichbleibenden Rahmenbedingungen die Zahl der inhaftierten Menschen, die nicht behandelt, schwer krank und weder krankheits- noch behandlungseinsichtig sind, hoch bleiben wird. Bei schweren Verhaltensstörungen mit Eigen- und Fremdgefährdung wird sich immer wieder die Frage einer Zwangsmedikation in Haft stellen. Zahlen zur Häufigkeit von psychiatrischen Zwangsmedikationen in Haft liegen jedoch ebenso

\footnotetext{
2 Dies bezieht sich vor allem auf die Entscheidungen des Bundesverfassungsgerichts in den Jahren 2011 (BVerfGE 128, 282 ff.; BVerfGE 129, $269 \mathrm{ff}$.) und 2013 (BVerfGE 133, $112 \mathrm{ff.}$.).
} 
wenig wie Leitlinien oder Empfehlungen für ihre Durchführung vor. Der vorliegende Artikel soll deshalb nach einer Einführung zur Versorgungssituation psychisch erkrankter Menschen in deutschen Gefängnissen auf die juristischen Voraussetzungen und geeignete Rahmenbedingungen für Zwangsmedikation in Haft eingehen.

\section{Die Versorgung psychisch erkrankter Menschen in Haft}

Ein großer Teil der psychisch erkrankten Menschen in Haft wird mithilfe von konsiliarisch tätigen Fachärzten für Psychiatrie und Psychotherapie in den Anstalten ambulant behandelt [5]. Diese psychiatrische Grundversorgung kann aufgrund eines Mangels an Psychiatern vor allem in Anstalten der ländlichen Regionen oft nicht ausreichend gewährleistet werden und es wird daher aktuell geprüft, ob in Zukunft telemedizinische Modellprojekte die langfristige ambulante Versorgung ergänzen können [20].

Wenn psychische Erkrankungen so schwer ausgeprägt sind, dass eine stationäre Behandlung notwendig wäre, fehlt für Menschen in Haft eine einheitliche Lösung. Anders als außerhalb der Gefängnisse, wo durch die regionale Pflichtversorgung psychisch erkrankte Menschen in Deutschland ein zeitnahes (bei Notfällen auch unmittelbares) stationäres Behandlungsangebot erhalten, gibt es für psychisch erkrankte Menschen in Haft keine Pflichtversorgungslösung [21]. Einige Länder (Baden-Württemberg, Bayern, Berlin, Brandenburg, Hessen, Niedersachsen, Nordrhein-Westfalen, Rheinland-Pfalz, Sachsen und Schleswig-Holstein) verfügen zwar über psychiatrische Abteilungen innerhalb des Justizvollzuges, allerdings gilt hier keine Aufnahmepflicht, sodass einerseits Wartelisten bestehen und andererseits keine stationäre Aufnahme erfolgen kann, wenn die Abteilungen voll belegt sind. Ein Vorhalten von Kapazitätsreserven für ungeplante Aufnahmen, wie in der regionalen Pflichtversorgung, findet in psychiatrischen Abteilungen des Justizvollzuges nicht statt. Andere Länder (z.B. Hamburg und Mecklenburg-Vorpommern) verlegen psychisch erkrankte
Menschen aus der Haft in eine Maßregelvollzugsklinik, wenn eine stationäre Behandlung notwendig wird, da diese Kliniken über hohe Sicherheitsstandards und die Möglichkeiten einer leitliniengerechten Behandlung verfügen. Hierfür sind allerdings freie Kapazitäten im Maßregelvollzug notwendig, da die Übernahme von psychisch erkrankten Strafgefangenen nicht deren primärer Versorgungsauftrag ist, die aber nicht immer gegeben sind. Angesichts der $\mathrm{Zu}$ nahme psychiatrischer Unterbringungen im Maßregelvollzug in den letzten Jahren [22] dürfte dieser Weg mancherorts komplizierter geworden sein. Dies kann dazu führen, dass lange Wartezeiten entstehen, bis eine störungsspezifische stationäre Behandlung begonnen werden kann. Eine weitere Möglichkeit zur leitliniengerechten Behandlung psychisch erkrankter Menschen ist die psychiatrische Behandlung in allgemeinpsychiatrischen Einrichtungen außerhalb des Justizvollzugs. Dies wird jedoch selten umgesetzt [23], was verschiedene Gründe hat, wie z.B. die als ungünstig angesehene Präsenz von Justizvollzugsbeamten auf allgemeinpsychiatrischen Stationen, die dort mindestens zu zweit für die Bewachung der Strafgefangenen während der Behandlung zuständig sind. Dass die Anwesenheit von Justizvollzugsbeamten auf akutpsychiatrischen Stationen bei psychotischen Patienten Ängste auslösen und deren Krankheitsverlauf beeinflussen kann, liegt nahe. Die letzte Möglichkeit, um eine leitliniengerechte Behandlung psychisch erkrankter Menschen in Haft zu ermöglichen, bietet die Haftunterbrechung gem. $\$ 455$ Strafprozessordnung (StPO), über die die zuständige Strafvollstreckungsbehörde entscheidet. Neben der Feststellung einer gesundheitsbedingten Vollzugsuntauglichkeit dürfen insbesondere auch Gründe der öffentlichen Sicherheit nicht entgegenstehen, was in der Praxis diesen Weg häufig versperrt [24]. Da als Rechtsfolge die bereits in Vollstreckung befindliche Strafhaft unterbrochen wird, stellt dieses Verfahren jedoch formal keine Behandlung von Strafgefangenen dar.

Diese Zusammenfassung der Versorgungsmöglichkeiten verdeutlicht, dass in weiten Teilen der Bundesrepublik psychisch erkrankten Menschen in Haft nicht verlässlich und zeitnah eine leitliniengerechte Therapie zuteilwird. Insbesondere bei schwer erkrankten Menschen, die aufgrund fehlender Krankheits- und Behandlungseinsicht eine ambulant empfohlene Therapie mit Psychopharmaka ablehnen, kann dies zu äußerst ungünstigen Verläufen führen, die mit Eigen- und Fremdgefährdung einhergehen. Vonseiten der Anstalten erfolgt dann in der Regel - wenn keine Verlegung in eine psychiatrische Abteilung möglich ist - eine Verlegung auf sogenannte Beobachtungs- und/oder besonders gesicherte Hafträume, die faktisch zu einer Isolation der Menschen führt. Manche dieser Räume sind mit Videokameras ausgestattet, sodass die Patienten überwacht werden können, was die Privatsphäre stark einschränkt. Vor allem Menschen mit psychotischen Störungen, Selbstverletzungen und Suizidalität werden so untergebracht und verbleiben mangels Alternativen und anhaltenden Gefährdungsaspekten gegebenenfalls für Monate in diesen Hafträumen. Für gewöhnlich führt dies $\mathrm{zu}$ einer Verschlechterung von psychopathologischen Symptomen, sodass es im Zeitverlauf vereinzelt zu schwergradigen Zustandsbildern mit Verwahrlosung, Denkzerfahrenheit und ausgeprägten inhaltlichen Denkstörungen der Patienten kommen kann. Eine Einwilligung zur Medikamenteneinnahme ist unter diesen Umständen meist nur noch schwer zu erreichen und auch die Möglichkeiten für psychotherapeutische und psychosoziale Interventionen sind stark eingeschränkt, da vor allem kontinuierliche Pflege durch ein multiprofessionelles Team fehlt. Dass diese Versorgung schwer erkrankter Patienten nicht dem normierten Äquivalenzprinzip ${ }^{3}$ - nach dem die medizinischen Leistungen im vollzuglichen Gesundheitswesen den Leistungen für gesetzlich Krankenversicherte gleichwertig sein müssen - entspricht, ist kaum zu bestreiten. Darüber hinaus können

\footnotetext{
3 Das auf Bundesebene in $\S 61$ StVollzG normierte Äquivalenzprinzip hat auch in den Landesvollzugsgesetzen seinen Niederschlag gefunden.
} 
Hier steht eine Anzeige.

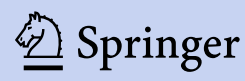


Nervenarzt 2021 · 92:27-35 https://doi.org/10.1007/s00115-020-00957-2

(c) Der/die Autor(en) 2020

\section{J. Fuß $\cdot$ I. Marquardt $\cdot$ P. Briken $\cdot$ N. Konrad}

\section{Zwangsmedikation psychisch erkrankter Menschen im Justizvollzug. Situation in Deutschland und Empfehlungen für die Praxis}

\section{Zusammenfassung}

Hintergrund. Medizinische Zwangsmaßnahmen stellen stets einen Eingriff in das Grundrecht auf körperliche Unversehrtheit sowie das Recht auf Selbstbestimmung des Patienten dar. Ist ein Patient inhaftiert, mithin ohnehin bereits in seinen Grundrechten stark eingeschränkt, und muss die Zwangsmaßnahme zudem in einer Justizvollzugsanstalt stattfinden, so stellt dies für alle Beteiligten insbesondere für den anordnenden Psychiater - eine besondere Herausforderung dar.

Ziel und Methode. Dieser Artikel soll die psychiatrische Versorgung psychisch erkrankter Menschen im Justizvollzug darstellen, die juristischen Voraussetzungen einer Zwangsmedikation erläutern und Empfehlungen für geeignete Rahmenbedingungen geben.
Ergebnisse und Diskussion. Für psychisch erkrankte Menschen in Haft gibt es in Deutschland keine Pflichtversorgungslösung entsprechend der Psychisch-Kranken-Gesetze, sodass diese bei schweren Erkrankungen nicht regelhaft einer stationär-psychiatrischen Versorgung zugeführt werden können. Dies kann zur Folge haben, dass bei ungünstigen Krankheitsverläufen eine Zwangsmedikation innerhalb des Justizvollzugs erwogen werden muss. Die Zwangsmedikation im deutschen Justizvollzug nimmt im Vergleich zur Zwangsmedikation in psychiatrischen Kliniken dabei eine Sonderstellung ein, da Ärzte in den meisten Bundesländern eine solche ohne richterliche Beteiligung anordnen können. Die Landesgesetzgeber sollten prüfen, ob hier eine gesetzliche Anpassung erforderlich oder sinnvoll wäre. Da die Umstände (rechtlich und versorgungstechnisch) für inhaftierte Patienten von denen in Kliniken stark abweichen, sollten die Rahmenbedingungen einer Zwangsmedikation wohl überlegt sein. Wir empfehlen das Hinzuziehen einer ethischen Fallberatung, wenn die Entscheidung ohne richterliche Beteiligung getroffen wird, sowie die Verlegung auf psychiatrische oder zumindest medizinische Krankenhausstationen zur Durchführung der Zwangsmedikation.

Schlüsselwörter

Gefängnispsychiatrie · Strafgefangene

Zwangsmaßnahmen · Zwangsbehandlung · Medizinethik

\section{Compulsory medication of people with mental disorders in the penal system. The situation in Germany and recommendations for clinical practice}

\section{Abstract}

Background. Coercive medical measures always represent an encroachment on the patient's basic right to physical integrity and right to self-determination. If a patient is imprisoned, his basic rights are already severely restricted, and the coercive measure must also take place in a correctional facility. This amounts to a special challenge for everyone involved-but especially for the psychiatrist in charge.

Objectives and Methods. This article is aimed at describing the psychiatric care of people with mental disorders in prisons, explaining the legal requirements for compulsory medication and giving recommendations for suitable conditions.

Results and Conclusions. In Germany, there is no legally regulated health-care solution for people with mental disorders in custody in accordance with the laws on the mentally ill of the German federal states (PsychischKranken-Gesetze). Therefore, in the case of serious mental disorders, imprisoned patients cannot be regularly provided with inpatient psychiatric care. As a result, compulsory medication within the correctional system must be considered if the course of the mental disorder is unfavorable. Compulsory medication in the German prison system has a special position compared with compulsory medication in psychiatric clinics, as doctors in most federal states can prescribe such medication without judicial involvement. The state legislators should check whether a legal adjustment would be necessary or helpful here. As the circumstances (legal and care-related) for imprisoned patients differ greatly from those of patients in clinics, the general conditions for compulsory medication should be carefully considered. We recommend consulting ethical case counseling if the decision is made without judicial involvement, and moving imprisoned patients to a psychiatric or at least to a medical ward before the administration of compulsory medication.

\section{Keywords}

Prison psychiatry · Inmates - Compulsory measures - Compulsory treatment $\cdot$ Medical ethics diese Zustände im Hinblick auf das Recht auf körperliche Unversehrtheit (neben einer Gefährdung für die Gesundheit der Patienten und bei bestehender Fremdgefährdung auch für das Personal) sowie im Hinblick auf eine menschenwürdige Unterbringung (bei andauernder Isolation) hochproblematisch sein. Außerdem werden die konsiliarisch tätigen Psychiater in eine Situation versetzt, in der sie sich aufgrund des dargestellten strukturellen Versorgungsproblems bei Nichthandeln jedenfalls fragen müssen, ob der Tatbestand einer unterlassene Hilfeleistung im Raum steht. An Handlungsoption bleibt ihnen jedoch häufig nur noch eine - ebenfalls stark in die Rechte des Patienten eingreifende Medikation gegen dessen Willen.

\section{Die juristischen Voraus- setzungen für Zwangs- medikation in Haft}

Gemäß \ 101 Strafvollzugsgesetz (StVollzG) sind Zwangsmaßnahmen auf dem Gebiet der Gesundheitsfürsorge nur bei Gefahr für das Leben oder schwerwiegender Gefahr für die $\mathrm{Ge}$ sundheit der Gefangenen zulässig, wenn 
diese krankheitsbedingt zur Einsicht in die Notwendigkeit der Behandlungsmaßnahmen nicht fähig sind, oder bei Gefahr für die Gesundheit Dritter. Bei Letzterem dürfte der Gesetzgeber eher an ansteckende Krankheiten und an eine dadurch bedingte Gefährdung von Besuchern, Bediensteten und/oder Mitgefangenen gedacht haben. Im Fall der psychiatrischen Erkrankung kann das Tatbestandsmerkmal der Gefährdung Dritter jedoch (ergänzend) eine Rolle spielen, wenn der psychisch erkrankte Mensch aggressives Verhalten an den Tag legt. Die Maßnahmen müssen nach der bundesgesetzlichen Regelung darüber hinaus für alle Beteiligten zumutbar sein, woran es beispielsweise fehlen kann, wenn ein Eingriff die Menschenwürde verletzt. ${ }^{4}$ Der Schwerpunkt dürfte in der Begründung der Verhältnismäßigkeit liegen: Die Maßnahme muss geeignet, erforderlich und im engeren Sinne angemessen sein, um als rechtmäßig zu gelten, und darf gemäß $\$ 101$ Absatz 3 StVollzG nur auf Anordnung und unter Leitung eines Arztes durchgeführt werden, außer es liegt eine lebensgefährliche Situation vor, die die Beteiligung eines Arztes nicht (mehr) zulässt.

Die Bundesländer haben eigene Regelungen getroffen, die gegenüber $₫ 101$ StVollzG teilweise weitergehende und differenzierte Voraussetzungen festlegen, was die Subsumtion im hier zu betrachtenden Fall der psychischen Erkrankung jedoch nicht einfacher macht. So dürften beispielsweise bestimmte Fälle der Zwangsernährung - jedenfalls von außen betrachtet - einfacher nachzuvollziehen sein, wenn Lebensgefahr besteht: Unterbleibt sie, droht der Tod des Gefangenen und mildere Mittel dürften nach Ablauf einer gewissen Zeit kaum ersichtlich sein. Bei einer psychischen Erkrankung macht bereits die Begründung einer Gesundheitsgefährdung Schwierigkeiten: Nach der kommentierenden Literatur zu $\$$ 101 StVollzG setzt die schwerwiegende Gefahr für die Gesundheit voraus, dass eine dauerhafte oder zumindest lang andauernde Beeinträchtigung wichtiger

${ }^{4}$ vgl. BeckOK Strafvollzug Bund/Wachs, 17. Ed.01.02.2018, StVollzG § 101 Rn. 4.
Körperfunktionen droht. ${ }^{5}$ Diese Definition ist auf psychopathologische Befunde nicht zugeschnitten. In Anlehnung an Zwangsbehandlungen nach Bürgerlichem Gesetzbuch (vgl. \$1906a BGB) wird mithin $\mathrm{zu}$ begründen sein, dass gewichtige gesundheitliche Nachteile mit der Maßnahme abgewendet werden können, die nicht zwingend organischer Natur sein müssen und bspw. in der Verhinderung einer Chronifizierung des Krankheitsbildes liegen können. Ist eine Chronifizierung bereits eingetreten, muss eine dem Patienten schadende erhebliche Verschlechterung des Gesundheitszustandes aufgrund weiterer Umstände begründet werden. Ergänzend wird sich in der Praxis der Psychiater auf die Gefährdung Dritter stützen können, soweit sich die psychische Erkrankung auch in aggressivem Verhalten äußert. Für sich alleine stehen kann dieses Tatbestandsmerkmal jedoch nicht, da dieser Gefährdung auch durch Isolation des psychisch erkrankten Menschen in besonders gesicherten oder Beobachtungshafträumen begegnet werden kann, mithin ein (vermeintlich) milderes Mittel zur Verfügung steht. In der Praxis ist die Isolation in der Regel ohnehin das zunächst gewählte Mittel, und dies grundsätzlich auch im Sinne der Verhältnismäßigkeit: Durch die Separierung und Beobachtung des psychisch erkrankten Menschen lässt sich das Risiko für Eigen- und Fremdgefährdung zunächst senken. Auch der Gesundheitszustand kann sich durch eine reizarme Umgebung in manchen Fällen verbessern. Diese Art der Unterbringung ist eine vollzugliche Maßnahme, keine medizinische, und erfolgt „lediglich“ unter Beteiligung eines Arztes. Dies gilt (außer in Baden-Württemberg) auch für die Fixierung, die ebenso - auf Zeit - die Selbstverletzung und Gefährdung Dritter ausschließen kann, aber selten zur Verbesserung des Gesundheitszustandes beitragen dürfte. Dauert eine isolierte Unterbringung über Wochen oder Monate ohne ersichtliche Zustandsverbesserung an, stellt sich folglich erneut die Frage der Behandlung: Der Psychiater

${ }^{5}$ vgl. BeckOK Strafvollzug Bund/Wachs, 17. Ed. 01.02.2018, StVollzG §101 Rn. 3. wird dabei vor die Aufgabe gestellt, nicht nur die Alternativlosigkeit (kein milderes Mittel), sondern auch den Erfolg der Behandlung vorauszusagen (Eignung). Beispielhaft kann dies beim Vorliegen einer akuten psychotischen Episode im Rahmen einer paranoiden Schizophrenie anhand der Studienlage und klinischer Erfahrungswerte erfolgen. Die zudem erforderliche Feststellung der fehlenden Einsichtsfähigkeit im Hinblick auf den Behandlungsbedarf dürfte sich in der Praxis beim Vorliegen schwerer psychischer Erkrankungen mit ausgeprägten Denkstörungen im Einzelfall aus dem psychopathologischen Befund ergeben. Auch sie ist aber eine erforderliche Voraussetzung und zu bejahen, sofern der psychisch erkrankte Mensch zur Einsicht in die Schwere seiner Krankheit und die Notwendigkeit von Behandlungsmaßnahmen oder zum Handeln gemäß solcher Einsicht krankheitsbedingt nicht fähig ist. ${ }^{6}$

Während deutschlandweit ein Richtervorbehalt die Entscheidung über Zwangsmedikation nach Maßregelvollzugs- und Psychisch-Kranken-Gesetzen in die Hände eines Gerichts legt, kann es im Vollzug dazu kommen, dass der Psychiater alleine über die Zwangsmedikation entscheidet, teilweise mit Zustimmung der Anstaltsleitung und/oder nach Einholung einer zusätzlichen ärztlichen Begutachtung. Nur in wenigen Bundesländern entscheidet (nach ärztlicher Stellungnahme) die Anstaltsleitung selbst und lediglich in Schleswig-Holstein und Baden-Württemberg erfolgt eine „echte“ Beteiligung des Gerichts. ${ }^{7}$ In den übrigen Ländern wird der Gefangene über die Möglichkeit, vorab eine gerichtliche Entscheidung herbeizuführen, aufgeklärt, und es wird eine angemessene Frist vor dem Vollzug der Maßnahme abgewartet, damit diese Möglichkeit genutzt werden kann. Nachdem die Regelungen

6 BVerfGE 128, 282 (304 f.).

7 Gemäß $\S 80$ Abs. 3 BWJVollzGB ist eine solche Maßnahme auf Antrag der JVA nur mit vorheriger richterlicher Entscheidung des Amtsgerichts zulässig; gemäß $§ 86$ Abs. 4 Ziffer 3 LStVollzG SH setzt eine ärztliche Zwangsmaßnahme voraus, dass das Gericht der Durchführung der Maßnahme nach Anhörung des Gefangenen zugestimmt hat. 
der Fixierungen im Justizvollzug eine Überarbeitung erfahren haben und eine gerichtliche Anordnung vorsehen, ist dieses Verhältnis fraglich. Da beide Vorschriften den Regelungen des unmittelbaren Zwanges und nicht denen der Gesundheitsfürsorge unterfallen, sollten die Landesgesetzgeber prüfen, ob eine Harmonisierung erforderlich, wenigstens aber sinnvoll ist. Die richterliche Entscheidung ist nach dem Bundesverfassungsgericht eine der Möglichkeiten, die der Ersetzung der fehlenden Zustimmung des Betroffenen dient. Ob mit oder ohne vorherige gerichtliche Beteiligung ist in allen Bundesländern die Maßnahme nachträglich gerichtlich überprüfbar, sodass eine Dokumentation zwingend erforderlich ist. Hilfreich kann es dabei sein, wenn Vollzug und Medizin unter Berücksichtigung datenschutzrechtlicher Belange und der ärztlichen Schweigepflicht gemeinsame Vordrucke erarbeiten, da vollzugliche und medizinische Anordnungen im Fall der Zwangsmedikation stets miteinander einhergehen. Dies gilt bereits dann, wenn vorher oder hinterher die Unterbringung in einem gesicherten (Beobachtungs-)Haftraum oder ggf. sogar eine Fixierung zwecks Durchführung der Maßnahme erforderlich ist. Die folgenden Empfehlungen zur Handhabung, wie die Beteiligung einer klinischen Ethikkommission, können dabei ebenso Teil einer strukturierten Dokumentation sein.

\section{Geeignete Rahmenbedingun- gen für Zwangsmedikation in Haft am Beispiel der Situation in Hamburg}

Im Hamburger Justizvollzug dürften Zwangsmedikationen in den letzten Jahren kaum eine praktische Bedeutung gehabt haben, da bisher schwer kranke Patienten in die hiesige forensischpsychiatrische Klinik verlegt werden konnten ${ }^{8}$, bis eine deutliche Zustandsbesserung oder Therapieadhärenz erreicht wurde. Anfang 2020 sprach diese Klinik einen Aufnahmestopp aus, da es zu einer

8 Entsprechend § 63 Hamburgisches Strafvollzugsgesetz erfolgte die Verlegung zum Zweck derBehandlung. deutlichen Zunahme von Unterbringungen nach $\$ 126 a$ StPO gekommen war und keine Aufnahmekapazität mehr bestand [25]. Durch die veränderte Versorgungslage kam es zu einer Zwangsmedikation bei einem inhaftierten Patienten mit paranoider Schizophrenie. Neben den oben beschriebenen juristischen Voraussetzungen stellte sich auch die Frage einer ethischen Abwägung und eines bestmöglichen Vorgehens. Als Ergebnis dieses Prozesses wurden die folgenden Empfehlungen für Zwangsmedikationen im Justizvollzug entwickelt:

\section{Abwägung des ethischen Dilemmas und Überprüfung der Indikation}

Die Zwangsmedikation von Menschen, deren Grundrechte bereits durch die Inhaftierung eingeschränkt sind, ist ein schwieriges medizinethisches Thema und gesellschaftlich besonders umstritten. Wenn eine psychiatrische Zwangsmedikation nicht auf einer psychiatrischen Krankenhausstation mit entsprechend geschultem Personal erfolgt, stellt dies ein Risiko dar. Zum Beispiel kann eine Zwangsmedikation im Vollzug traumatisierender als in einem Krankenhaus erlebt werden, da die Prozedere weniger auf die Bedürfnisse von psychiatrischen Patienten abgestimmt sind. Die Behandler befinden sich in einer klassischen Dilemmasituation, in der sie zwischen zwei ungünstigen Szenarien wählen müssen (Zwangsmedikation im Vollzug vs. Nichthandeln), weil das erwünschte Szenario (Verlegung auf eine psychiatrische Station zur Durchführung der Zwangsmedikation) aufgrund unzureichender Versorgungsbedingungen häufig nicht möglich ist. Obwohl die Behandler die Versorgungsbedingungen in der Regel kaum beeinflussen können, müssen sie das ärztliche Vorgehen unter diesen Bedingungen verantworten. Die ausführliche ethische Abwägung einer Zwangsmedikation erfolgt außerhalb des Justizvollzugs im Rahmen der Begutachtung und der richterlichen Entscheidung. In den Bundesländern, in denen keine gerichtliche Beteiligung bei Zwangsmedikation im Justizvollzug erfolgt, sollte diese ethische Abwägung anders orga- nisiert werden. Ähnlich wie bei anderen weitreichenden medizinischen Entscheidungen, empfiehlt sich in diesem Fall eine ethische Fallberatung, um die möglichen Szenarien abzuwägen. In den Hamburger Anstalten, die vom Universitätsklinikum Hamburg-Eppendorf psychiatrisch versorgt werden, tritt eine interdisziplinäre klinische Ethikkommission zusammen, die den Fall erörtert und ethisch aus verschiedenen Blickwinkeln beleuchtet. Außerdem muss - jedenfalls in Hamburg - eine ärztliche Zweitsicht erfolgen, die juristisch notwendig, aber auch für die indizierende Person entlastend sein kann. Dies gilt auch für die in Hamburg erforderliche Zustimmung der Anstaltsleitung. Für konsiliarisch tätige Psychiater in Gefängnissen, die nicht im Umfeld einer größeren Klinik arbeiten, sollten vergleichbare Strukturen geschaffen werden, in denen die wichtigsten Positionen und Argumente im Vorfeld einer geplanten Zwangsmedikation von einem möglichst diversen Expertengremium (z.B. Ärzte, Ethiker, Juristen, Seelsorger, Sozialarbeiter u. a.) erörtert werden können. Hier kann auch die komplizierte Rolle der Konsiliarpsychiater reflektiert werden, die sich in einem Loyalitätskonflikt zwischen Patient und Anstalt befinden können.

\section{Schaffung geeigneter Umgebungsbedingungen}

Beobachtungs- und besonders gesicherte Hafträume einer Anstalt sind keine geeignete Umgebungsbedingung für eine Zwangsmedikation von psychisch erkrankten Menschen. Die Vermeidung einer traumatisierenden Erfahrung muss auch in Hinblick auf zukünftige Compliance bei der Durchführung hohe Priorität haben - die Behandler müssen den Prozess steuern können. Bei einer Durchführung auf einer Station des Vollzugs wäre zu befürchten, dass z.B. bei körperlicher Auseinandersetzung ein sogenannter HSP(HelmSchild-Pfefferspray)-Einsatz zur Sicherung des Patienten und anschließender Fixierung durch den Vollzug angeordnet und vollzogen wird. Dieser Ablauf kann jedoch so schwer traumatisierend sein, dass er die positiven Effekte einer Me- 
Hier steht eine Anzeige.

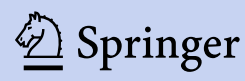


dikation überwiegen würde. Ein solche Form der Eskalation muss unbedingt verhindert werden und sollte im Vorfeld offen mit den Akteuren besprochen werden - bei drohender Eskalation kann die Zwangsmedikation auch abgebrochen und zu einem späteren Zeitpunkt mit günstigeren Bedingungen erneut vollzogen werden. Wir empfehlen daher generell die Verlegung von Patienten auf psychiatrische, zumindest medizinische Krankenhausstationen innerhalb des Justizvollzugs. Diese sind aus ärztlicher Perspektive besser geeignet, da dort auch seltene Nebenwirkungen, wie z.B. Anaphylaxie bei erstmaliger Gabe eines Depotpräparats frühzeitig diagnostiziert und behandelt werden können. Außerdem ist es gegenüber den Patienten ein wichtiges Signal, dass die Maßnahme im klinischen Kontext stattfindet, um zu markieren, dass es sich um eine ärztliche Maßnahme (nicht um Strafe o. Ä.) handelt, auch wenn diese juristisch den Regelungen des unmittelbaren Zwanges und nicht denen der Gesundheitsfürsorge zuzuordnen ist. Es ist wichtig, das Personal dieser Stationen frühzeitig in den Prozess einzubeziehen. In Hamburg soll dies z.B. bereits durch einen teilnehmenden Vertreter im Rahmen der klinischen Ethikkommission erfolgen. Es sollte ferner geprüft werden, ob zur Durchführung einer Zwangsmedikation auf eine Fixierung, die eine hohe Belastung darstellt, verzichtet werden kann. Ein kurzes Festhalten der Patienten kann genügen, um eine Depotmedikation $\mathrm{zu}$ verabreichen. Wenn eine Zwangsmedikation im Vollzug angeordnet wird, sind die Patienten häufig bereits seit längerer Zeit in einem Beobachtungs- oder besonders gesicherten Haftraum untergebracht. Es kann Vollzugsbeamte geben, die sich im Rahmen der Unterbringung um ein besonders vertrauensvolles und gutes Verhältnis zu den psychisch erkrankten Menschen bemühen (vergleichbar mit einer Art Bezugspflege in der Psychiatrie). Es kann hilfreich sein, wenn diese die Maßnahme begleiten. Auch diesbezüglich sind ein enger Austausch und ein Konsens mit den Beteiligten der Anstalt erforderlich und empfehlenswert. Darüber hinaus sollten die Empfehlungen der aktuellen
S3-Leitlinie „Verhinderung von Zwang: Prävention und Therapie aggressiven Verhaltens bei Erwachsenen" beachtet werden. In Hamburg konnte durch diese Maßnahmen (insbesondere die Verlegung des Patienten in das Vollzugskrankenhaus und die Hinzuziehung von psychiatrisch versiertem Vollzugspersonal) letztlich eine drohende Eskalation verhindert werden, sodass die erfolgte Zwangsmedikation des Patienten nach Abklingen der Symptome von ihm als hilfreich eingeschätzt wurde.

\section{Fazit für die Praxis}

- Indikationen einer Zwangsmedikation unter Haftbedingungen gehören zu den Herausforderungen an die Psychiatrie und Medizinethik.

- Anders als außerhalb des Justizvollzugs, wo ein Richtervorbehalt besteht, wird bei Zwangsmedikation von inhaftierten Menschen in den meisten Bundesländern die Entscheidung von einem Arzt (nach vorheriger Beteiligung der Anstaltsleitung oder eines weiteren Arztes) getroffen. Die Landesgesetzgeber sollten prüfen, ob hier eine gesetzliche Anpassung erforderlich oder sinnvoll wäre.

- Die Verhaltensspielräume und Grundrechte inhaftierter Patienten sind von vornherein eingeschränkt. Die Rahmenbedingungen einer Zwangsmedikation sollten daher wohlüberlegt und mit der Anstalt abgesprochen sein.

- Der zunehmende Abbau allgemeinpsychiatrischer Betten und die Verkürzung der Liegezeiten haben möglicherweise dazu beigetragen, dass einzelne psychisch erkrankte Menschen nicht behandelt, obdachlos und kriminell werden [26]. Daher wird das Thema einer Zwangsmedikation in Haft auch zukünftig eine hohe Relevanz haben.

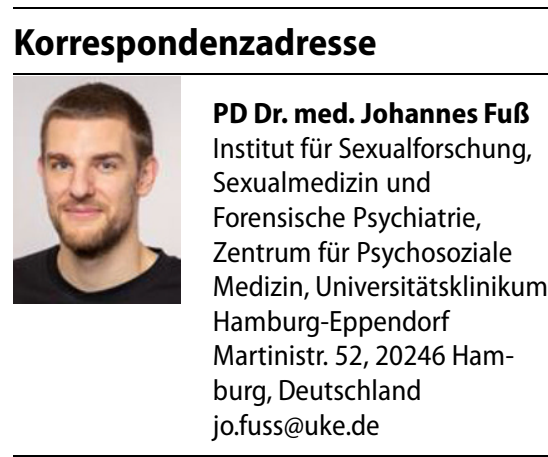

Danksagung. Wir danken Prof. Dr. med. Tilman Steinert, Dr. Katharina Woellert und den Mitgliedern der ethischen Fallberatung am UKE Hamburg für ihre Unterstützung und Überlegungen zur Zwangsmedikation im Justizvollzug.

Funding. Open Access funding provided by Projekt DEAL.

\section{Einhaltung ethischer Richtlinien}

Interessenkonflikt. J. Fuß, I. Marquardt, P. Briken und N. Konrad geben an, dass kein Interessenkonflikt besteht.

Für diesen Beitrag wurden von den Autoren keine Studien an Menschen oder Tieren durchgeführt. Für die aufgeführten Studien gelten die jeweils dort angegebenen ethischen Richtlinien.

Open Access. Dieser Artikel wird unter der Creative Commons Namensnennung 4.0 International Lizenz veröffentlicht, welche die Nutzung, Vervielfältigung, Bearbeitung, Verbreitung und Wiedergabe in jeglichem Medium und Format erlaubt, sofern Sie den/die ursprünglichen Autor(en) und die Quelle ordnungsgemäß nennen, einen Link zur Creative Commons Lizenz beifügen und angeben, ob Änderungen vorgenommen wurden.

Die in diesem Artikel enthaltenen Bilder und sonstiges Drittmaterial unterliegen ebenfalls der genannten Creative Commons Lizenz, sofern sich aus der Abbildungslegende nichts anderes ergibt. Sofern das betreffende Material nicht unter der genannten Creative Commons Lizenz steht und die betreffende Handlung nicht nach gesetzlichen Vorschriften erlaubt ist, ist für die oben aufgeführten Weiterverwendungen des Materials die Einwilligung des jeweiligen Rechteinhabers einzuholen.

Weitere Details zur Lizenz entnehmen Sie bitte der Lizenzinformation auf http://creativecommons.org/ licenses/by/4.0/deed.de.

\section{Literatur}

1. Penrose LS (1939) Mental disease and crime: outline of a comparative study of European statistics. Br J Med Psychol 18(1):1-15

2. Mundt AP et al (2015) Psychiatric hospital beds and prison populations in South America since 1990: Does the Penrose hypothesis apply? JAMA 72(2):112-118 
3. Chow WS, Priebe S (2016) How has the extent of institutional mental healthcare changed in Western Europe? Analysis of data since 1990. BM Open. https://doi.org/10.1136/bmjopen-2015010188

4. Priebe $S$ et al (2005) Reinstitutionalisation in mental health care: comparison of data on service provision from six European countries. BMJ 330(7483):123-126

5. Konrad N (2007) Psychiatrische Konsiliartätigkeit im deutschen Justizvollzug. Psychosom Konsiliarpsychiatr 1(3):216-219

6. Lamb HR, Weinberger LE (2005) The shift of psychiatric inpatient care from hospitals to jails and prisons. J Am Acad Psychiatry Law 33(4):529-534

7. Sisti DA, Segal AG, Emanuel EJ (2015) Improving long-term psychiatric care: bring back the asylum JAMA 313(3):243-244

8. Lamb HR (2015) Does deinstitutionalization cause criminalization? The penrose hypothesis. JAMA 72(2):105-106

9. Konrad N (2002) Prisons as new asylums. Curr Opin Psychiatry 15(6):583-587

10. Zinkler M (2008) Früher entlassen - schneller im Maßregelvollzug? Zum Verhältnis von allgemeiner und forensischer Psychiatrie. Recht Psychiatr 26(2):102-105

11. Fazel S, Danesh J (2002) Serious mental disorder in 23000 prisoners: a systematic review of 62 surveys. lancet 359(9306):545-550

12. Fazel S et al (2016) Mental health of prisoners: prevalence, adverse outcomes, and interventions. LancetPsychiatry 3(9):871-881

13. Von Schönfeld CE et al (2006) Prävalenz psychischer Störungen, Psychopathologie und Behandlungsbedarf bei weiblichen und männlichen Gefangenen. Nervenarzt 77(7):830-841

14. Schildbach S, Schildbach C (2018) Criminalization through transinstitutionalization: a critical review of the Penrose hypothesis in the context of compensation imprisonment. Front Psychiatry 9:534

15. Forschungsnetzwerk Wohnungslosigkeit und Gesundheit an der Charité (2020) Zählung wohnungsloser Menschen in psychiatrischen Krankenhäusern. https://wohnungslosigkeitgesundheit.charite.de/forschung/projekte/ zaehlung_wohnungsloser_menschen_in_ psychiatrischen_krankenhaeusern/.Zugegriffen: 19. Apr. 2020

16. Statistisches Bundesamt (2019) Rechtspflege: Strafvollzug - Demographische und kriminologische Merkmale der Strafgefangenen zum Stichtag 31.3. Fachserie 10, Reihe 4.1

17. Statistisches Bundesamt (2020) In der Strafverfolgungsstatistik erfasste Personen mit Untersuchungshaft. https://www.destatis.de/ DE/Themen/Staat/Justiz-Rechtspflege/Tabellen/ erfasste-personen-uhaft.html. Zugegriffen: 19. Apr. 2020

18. Flammer E, Steinert T (2015) Involuntary medication, seclusion, and restraint in German psychiatric hospitals after the adoption of legislation in 2013. Front Psychiatry 6:153

19. Flammer E, Steinert T (2015) Auswirkungen der vorübergehend fehlenden Rechtsgrundlage für Zwangsbehandlungen auf die Häufigkeit aggressiver Vorfälle und freiheitseinschränkender mechanischer Zwangsmaßnahmen bei Patienten mit psychotischen Störungen. Psychiat Prax 42(05):260-266

20. Lehmann M (2019) Erfahrungen eines substituierenden Anstaltsarztes. Vortrag auf dem
Symposium „Substitutionsbehandlung Opiatabhängiger in Haftanstalten" auf dem DGPPN Kongress 2019

21. Konrad N (1997) Psychiatrie im Justizvollzug. Recht Psychiatr 15:51-59

22. Statistisches Bundesamt (2015) Strafvollzugsstatistik: Im psychiatrischen Krankenhaus und in derEntziehungsanstalt aufgrund strafrichterlicher Anordnung Untergebrachte (Maßregelvollzug) 2013/2014. Wiesbaden

23. Konrad N, Missoni L (2001) Psychiatrische Behandlung von Gefangenen in allgemeinpsychiatrischen Einrichtungen am Beispiel von NordrheinWestfalen und Rheinland-Pfalz. Psychiatr Prax 28(01):35-42

24. KonradN (2000) Psychisch Krankeim Justizvollzug Sicht des forensischen Psychiaters. Z Arztl Fortbild Qualitatssich 94(4):288-291

25. Knecht, G (2020) Vortrag am 30. Jan. 2020 in der Asklepios Klink Nord-Ochsenzoll, Hamburg

26. Fuller Torrey E (2015) Deinstitutionalization and the rise of violence. CNS Spectr 20(3):207-214. https://doi.org/10.1017/S1092852914000753
GRATIS

Ein Service für Autoren von Springer Medizin N

Fortbildungen für Autor*innen und Gutachter*innen

Die ersten Veröffentlichungen sind für Jeden ein wichtiger Schritt in der angestrebten Karriere. Wissenschaftliche Artikel sind entscheidend dafür, dass die eigene Arbeit in der Community wahrgenommen wird. Es geht darum, die eigenen Ideen national und international auszutauschen und sicherzustellen, dass die Ergebnisse Wirkung erzielen.

Die Online-Kurse der Autorenwerkstatt helfen, sich leicht einen Überblick über das Schreiben, Einreichen, Begutachten und Veröffentlichen eines Manuskripts zu verschaffen.

5 Online-Kurse zu den wichtigsten Standards des wissenschaftlichen Publizierens:

- Wie verfasse ich ein Manuskript?

- Writing in English für deutschsprachige Autorinnen und Autoren

- Wie funktionieren Publikation und Begutachtung?

- Anleitung zur Open-AccessVeröffentlichung

- Leitfaden zur Peer-ReviewBegutachtung

Für alle, die auf SpringerMedizin.de registriert sind!

Jetzt gratis fortbilden unter www.springermedizin.de/ autorenwerkstatt/ 